\title{
\begin{tabular}{l|l} 
Mitraries & DSpace@MIT
\end{tabular}
}

\author{
MIT Open Access Articles
}

\section{Statistical biases and errors inherent in photoclinometric surface slope estimation with natural light}

The MIT Faculty has made this article openly available. Please share how this access benefits you. Your story matters.

Citation: Bertsatos, loannis, and Nicholas C. Makris. “Statistical Biases and Errors Inherent in Photoclinometric Surface Slope Estimation with Natural Light." Icarus 208, no. 2 (August 2010): 798-810.

As Published: http://dx.doi.org/10.1016/j.icarus.2010.03.019

Publisher: Elsevier

Persistent URL: http://hdl.handle.net/1721.1/105422

Version: Author's final manuscript: final author's manuscript post peer review, without publisher's formatting or copy editing

Terms of use: Creative Commons Attribution-NonCommercial-NoDerivs License 


\title{
Statistical Biases and Errors Inherent in Photoclinometric Surface Slope Estimation with Natural Light
}

\author{
Ioannis Bertsatos ${ }^{*, a}$, Nicholas C. Makris*,a \\ ${ }^{a}$ Massachusetts Institute of Technology, Cambridge, Massachusetts 02139
}

Abstract

Photoclinometry is the most common method used to obtain high-resolution topographic maps of planetary terrain. We derive the likelihood function of photoclinometric surface slope from (1) the probability distribution of the measured photon count of natural sunlight through a Charge-Coupled Device (CCD) including uncertainty due to camera shot noise, camera read noise, small scale albedo fluctuation and atmospheric haze, and (2) a photometric model relating photocount to surface orientation. We then use classical estimation theory to determine the theoretically exact biases and errors inherent in photoclinometric surface slope and show when they may be approximated by asymptotic expressions for sufficiently high sample size. We show how small-scale albedo variability often dominates biases and errors, which may become an order of magnitude larger than surface slopes when surface reflectance has a weak dependence on surface tilt. We provide bounds on the

\footnotetext{
*Corresponding author

**Principal corresponding author

Email addresses: ibertsat@mit.edu (Ioannis Bertsatos), makris@mit.edu (Nicholas C. Makris)
} 
minimum possible error of any unbiased photoclinometric surface slope estimate, and compute the sample sizes necessary to constrain errors within desired design thresholds.

Key words: Photometry 


\section{Introduction}

High-resolution elevation maps of planetary terrain are typically obtained by the method of photoclinometry (e.g. Malin et al., 1992; McEwen et al., 2003; Kirk et al., 2003b; Schenk, 2005), which relates variations in surface radiance to variations in surface orientation relative to the light source, typically the Sun, and the optical receiver, typically on a spacecraft (Davis and Soderblom, 1984; McEwen, 1991; Kirk et al., 2003a). While other methods also exist to produce topographic models, including stereogrammetry and radar altimetry, photoclinometry offers significant advantages since it (1) requires only a single image, and (2) can provide higher resolution measurements (McEwen, 1991).

It has been observed, however, that photoclinometry may not work very well under certain lighting conditions that provide little topographic contrast, and that these conditions typically correspond to small incidence angles (e.g. Davis and McEwen, 1984; Efford, 1991; Jankowski and Squyres, 1991; Kirk et al., 2003a,b). Uncertainties in surface albedo may also lead to errors in surface slope estimates that are significant for small-scale albedo variations (Howard, 1982; Kirk et al., 2003b), but become relatively insignificant for large-scale albedo variations (e.g. Beyer et al., 2003).

The primary purpose of the present paper is to provide a formulation of uncertainties and analysis of errors that (1) is consistent with the behavior of the likelihood function (Fisher, 1956) of the photoclinometric surface slope estimate that governs the uncertainties, and (2) accounts for all the primary photoclinometric error sources, including albedo, haze, camera read noise and camera shot noise, in a unified manner. Here, classical estimation 
theory (Fisher, 1956; Kay, 1993) is used to provide a method for determining both the exact and asymptotic biases and errors inherent in a Maximum Likelihood Estimate (MLE) of photoclinometric surface slope given the probability distribution of the measured Charge-Coupled Device (CCD) data and the nonlinear physical model relating the measured CCD data to surface slope by planetary surface reflectance (e.g. McEwen, 1991). The formulation also provides bounds on the minimum possible error for any unbiased photoclinometric estimate of surface slope as well as necessary conditions on sample size to attain this error bound, or a desired design threshold on error. The asymptotic biases and errors are determined by series expansion in inverse orders of sample size, where higher order terms vanish in decreasing order as uncertainty decreases until the Cramer-Rao Lower Bound (CRLB) or firstorder error term is attained (Naftali and Makris, 2001). Since approximations to investigate photoclinometric errors (e.g. Davis and McEwen, 1984; Efford, 1991; Jankowski and Squyres, 1991; Beyer et al., 2003; Kirk et al., 2003a,b) have previously not been formulated in terms of the likelihood function that governs uncertainties, error bounds, asymptotic behavior for decreasing uncertainty, necessary sample sizes, and exact theoretical biases and variances have not been previously provided. We show that in many practical photoclinometric scenarios the approximate asymptotic biases and errors for a single sample differ dramatically from the exact ones, making asymptotic expressions for errors applicable only when a large number of independent samples is available. Moreover, the asymptotic expressions for errors must be formulated in terms of the likelihood function as in Shenton and Bowman (1977); Barndorff-Nielsen and Cox (1994); McCullagh (1987); Naftali 
and Makris (2001) for them to properly converge as uncertainty decreases or sample size increases.

In Section 2 we derive the likelihood function, the MLE and biases and errors for photoclinometric surface estimation. The MLE is chosen because it is known to become asymptotically unbiased and attain the minimum possible mean square error of any unbiased estimate as sample size becomes large or uncertainty becomes small (Rao, 1966; Fisher, 1956). In Section 3 we compute the exact theoretical biases and root mean square errors of the surface slope MLE for various photometric functions and typical values of camera read noise, camera shot noise, atmospheric haze, and albedo variability. We show that the biases and root mean square errors grow rapidly when the dependence of measured intensity on surface slope approaches a constant, and that albedo variability is typically the dominant source of biases and errors. We also present estimation methods for minimizing these biases and errors to obtain surface slope estimates that fall within desired design error thresholds.

\section{The Likelihood Function and Maximum Likelihood Estimation of Planetary Surface Slopes}

In photoclinometry, natural light from a thermal source, such as the Sun or a star, typically acts as the source of planetary surface illumination. Natural light is known to undergo Circular Complex Gaussian Random (CCGR) field fluctuations and exponentially distributed instantaneous intensity fluctuations, as a consequence of the central limit theorem (Goodman, 1985, chap. 4). Spacecraft observations of planetary surfaces are typically made 
with photon-counting CCD cameras (e.g. Malin and Edgett, 2001; McEwen et al., 2003), where the number of detected photons is known to follow the conditional Poisson probability distribution for a given average light intensity. Since the average intensity of natural light follows a Gamma distribution, conditional integration over all possible intensities leads to the negative binomial distribution for the photocount (Goodman, 1985, chap. 9).

Photocount is related to planetary surface orientation by modeling the reflectance properties of the planetary surface with a photometric function. Many planetary surfaces have been successfully modeled with one or a combination of a such closed-form empirical functions, including Lambert's law, Minnaert's law, and the lunar-Lambert model (McEwen, 1991).

In this section, we discuss three common photometric functions used to model planetary surface reflectance. We then use classical estimation theory to derive the likelihood function and MLE for photometric surface slope estimation, the theoretical lower bound on surface slope error, and necessary conditions on sample size to appropriately constrain biases and errors within desired design error thresholds.

\subsection{Photometric Functions of Planetary Surface Reflectance}

The most commonly used photometric function in planetary topography applications is the lunar-Lambert function first introduced by McEwen (1986),

$$
I\left(\mu_{n}, \mu_{0 n}, \alpha\right)=B o(\alpha)\left[\frac{2 L(\alpha) \mu_{0 n}}{\mu_{n}+\mu_{0 n}}+(1-L(\alpha)) \mu_{0 n}\right]
$$

where $I\left(\mu_{n}, \mu_{0 n}, \alpha\right)$ is the reflectance function, $\mu_{n}=\cos \epsilon_{n}, \mu_{0 n}=\cos \iota_{n}$, and $\epsilon_{n}, \iota_{n}$ are the emission and incidence angles respectively, as shown in Fig. 1. 
The phase angle $\alpha$ corresponds to the angle between the incidence and emission angles, and $B o(\alpha)=I(1,1, \alpha)$ is defined as the intrinsic albedo. $L(\alpha)$ is the ratio of the lunar to the Lambertian component in the lunar-Lambert function, so that in the limit $L(\alpha) \rightarrow 0$ the modeled surface is Lambertian, while in the limit $L(\alpha) \rightarrow 1$, the surface is lunar. The photometric function is the ratio of the intensity incident at angle $\iota_{n}$ to that reflected to the receiver at emission angle $\epsilon_{n}$.

\section{[Figure 1]}

For many planetary surfaces and phase angles, $L(\alpha)$ can be well approximated as a constant $L$, especially when observations are made over a limited range of incidence angles. Beyer et al. (2003), for example, show that variations in $L$ for Martian terrain lead to small errors of $10 \%$ of the $L=0.55$ mean for Mars Orbiter Camera (MOC) (Malin and Edgett, 2001) incident angles in the vicinity of $25-45^{\circ}$. Similarly, the effect of large-scale albedo variations, i.e. changes in the value of $B o(\alpha)$ across the planetary terrain, can be minimized by scaling out the average brightness of the imaged region (Beyer et al., 2003). Small-scale variations in albedo cannot be similarly accounted for and may lead to much larger errors (Howard, 1982; Kirk et al., 2003b; Beyer et al., 2003). Here we model $B o(\alpha)$ as a Gaussian random variable based on a central limit theorem assumption of many independent sources of albedo variation. The mean is set to the average albedo value across the imaged region and the standard deviation is defined as proportional to a fraction of the mean following calculations presented by Bell et al. (2008) for typical Martian surfaces.

The illumination and zenith direction vectors define the principal plane 
(e.g. Ranson et al., 1991, Fig. 1). It is common in planetary applications for satellite cameras to be close to nadir-looking, so that the difference between the emission angle and its projection on the principal plane is negligible. Assuming that local surface slopes are always in the up- or down-sun direction, which is also the direction where reflectance is most sensitive to slope changes for a Lambertian surface or small emission angles in the lunar-Lambert model of Eq. 1 (e.g. Beyer et al., 2003; Kirk et al., 2003b), the emission and incidence angles can then be written in terms of slope $\theta$ with respect to a flat surface, $\epsilon_{n}=\epsilon_{z}-\theta$ and $\iota_{n}=\iota_{z}-\theta$. Here $\iota_{z}$ and $\epsilon_{z}$ are defined as the known angles that the incident and emission directions make to the zenith direction, respectively.

With these assumptions, the photometric function can be written as

$$
I\left(\mu_{n}, \mu_{0 n}, \alpha\right) \equiv I(\theta)=\operatorname{Bof}(\theta)
$$

where

$$
f(\theta)=\left[L \frac{\cos \left(\iota_{z}-\theta\right)}{\cos \left(\frac{\iota_{z}+\epsilon_{z}}{2}-\theta\right) \cos \left(\frac{\iota_{z}-\epsilon_{z}}{2}\right)}+(1-L) \cos \left(\iota_{z}-\theta\right)\right]
$$

Surface slope $\theta$ can be estimated from knowledge of $I(\theta)$. While surface slopes will be underestimated if their azimuth does not lie in the principal plane, this error is found to be negligibly small for relatively flat topography, as are errors introduced when the satellite viewing direction is off the principal plane (Beyer et al., 2003).

Equation 3 is plotted as a function of surface slope $\theta$ and incident angle with respect to flat topography $\iota_{z}$ for the parameter $L$ set to 0,1 , and 0.55 in Figs. 2(a), 3, and 4(a), respectively. The case $L=0.55$ is shown here 
as an appropriate choice for Martian terrain (Beyer et al., 2003). For other planetary bodies, McEwen (1991) provides best-fit $L(\alpha)$ values for various terrain types. The angle of emission with respect to the $z$-axis is assumed to be $\epsilon_{z} \approx 0^{\circ}$, which is equivalent to the typical case of a nadir-looking satellite, so that the true emission angle is $\epsilon_{n}=-\theta$. In all three figures, white dashed lines highlight where the derivative of $I$ with respect to $\theta$ is zero so that the dependence of the CCD measurement on surface slope is constant. White dot-dashed lines correspond to the direction of specular reflection, which in this case occurs when $\iota_{n}=-\epsilon_{n}=\theta$, or equivalently $\iota_{z}=\iota_{n}+\theta=2 \theta$. Finally, black lines denote lines of constant true incidence angle, $\iota_{n}$, which are described by the equation $\iota_{z}=\theta+\iota_{n}$, so that their slope and $y$-intercept are 1 and $\iota_{n}$, respectively.

\section{[Figure 2, Figure 3, Figure 4]}

The Lambertian photometric function of Eqs. 2-3 for $L=0$ is symmetric about the line where the true incidence angle $\iota_{n}$ equals zero, which is also where $\mathrm{d} I / \mathrm{d} \theta$ is zero, as a consequence of Lambert's cosine law, and as can be seen in Fig. 2(a). The lunar photometric function of Eqs. 2-3 for $L=1$ is instead antisymmetric about the direction of specular reflection, while its derivative with respect to surface slope goes to zero when the incident and emission directions become collinear, as can be seen in Fig. 3(a). For the lunar-Lambert photometric function of Eqs. 2-3 for $L=0.55$, the $\mathrm{d} I / \mathrm{d} \theta=0$ curve (white dashed line) is close to the $\iota_{n}=0$ line for small tilt angles $\theta$, while it gradually moves towards the $\iota_{z}=0$ line as $\theta$ increases, as can be seen in Fig. 4(a). For the Martian example of $L=0.55$, and for small tilt angles, the lunar-Lambert surface then approaches Lambert's cosine law, but 
becomes similar to a lunar surface as the surface slopes become larger.

The Lambertian, lunar and lunar-Lambert photometric functions are also plotted as functions of the true incidence angle $\iota_{n}=\iota_{z}-\theta$, for different values of the angle between the illumination direction and the zenith direction, $\iota_{z}$, in Figs. 2(b)-4(b). These plots are constructed by cutting along the white dotted lines of Figs. 2(a)-4(a) from right to left. Again, we note that the Lambertian photometric function depends only on the value of the true incidence angle $\iota_{n}$, while the lunar photometric function becomes independent of surface slope when the incident and emission directions are collinear.

\subsection{The Probability Distribution of CCD Photocount Measurements of Plan-} etary Surface Reflectance

Charge-Coupled Devices (CCDs) typically form the basic recording unit of the high-performance cameras used for space exploration missions (e.g. Malin and Edgett, 2001; McEwen et al., 2003) by measuring the number of electrons released from a photosurface when an electromagnetic field is incident upon it. This number is linearly proportional to the number of incident photons, which in turn is a function of the average light intensity incident on the photosurface (Janesick, 2001, chap. 6), so that the CCD output signal can be parameterized in terms of average intensity.

Natural light from thermal sources, such as the Sun, is known to follow Circular Complex Gaussian Random (CCGR) field fluctuations by the central limit theorem, so that average intensity is described by the Gamma distribution (Goodman, 1985, chap. 9). Since the number of photon arrivals for a given light intensity is known to be a Poisson random variable, the statistics of CCD-measured photocount then follow the negative binomial 
distribution (Goodman, 1985, chap. 9). For thermal light at optical frequencies, and for the common integration times of CCDs (e.g. Malin and Edgett, 2001), the discrete negative binomial distribution can be well approximated by the continuous Gaussian probability density (see Appendix A),

$$
P_{K}(K \mid \theta)=\frac{1}{\sqrt{2 \pi} \sigma_{K}(\theta)} \exp \left(-\frac{1}{2}\left[\frac{K-\bar{K}(\theta)}{\sigma_{K}(\theta)}\right]^{2}\right)
$$

where $K$ is the measured photocount.

The mean and variance of $K$ have been derived in Appendix A (Eqs. 34, 35) and are repeated here for convenience

$$
\begin{aligned}
\bar{K} & =\gamma[I(\theta)+H]=\gamma[\overline{B o} f(\theta)+H] \\
\sigma_{K}^{2} & =\bar{K}+\bar{K}^{2} \sigma_{B o}^{2} / \overline{B o}^{2}+\sigma_{R}^{2}
\end{aligned}
$$

where $\gamma$ is a known proportionality constant that depends on incident solar flux, camera integration time, pixel surface area and other parameters as described in Eq. 20, $\overline{B o}, \sigma_{B o}^{2}$ are the mean and variance of surface albedo, respectively, and $H$ is the expected intensity of atmospheric haze which is assumed to be a known constant (e.g. Beyer et al., 2003). Atmospheric haze is described by a CCGR field that is independent and additive to the CCGR field scattered from the surface which carries reflectance information. The variances or expected intensities of these two fields then add, so that the haze contribution increases the mean and variance of the photocount $K$. This leads to a dilution of surface reflectance information in the total photocount. Atmospheric haze often contributes minimally to topographic shading (e.g. Beyer et al., 2003). Kirk et al. (2001) provide a model for how haze is affected by changes in atmospheric conditions and illumination geometry. 
The photocount variance then has signal-independent components due to camera read noise $\sigma_{R}^{2}$ (Janesick, 2001, chap. 7), and atmospheric haze $\sigma_{\text {haze }}^{2} \equiv \gamma H$, and signal-dependent components $\gamma \overline{B o} f(\theta)$ for shot noise and $\bar{K}^{2} \sigma_{B o}^{2} / \overline{B o}^{2}$ for albedo uncertainty. The signal-dependent components arise from the Poisson nature of photon statistics, the CCGR fluctuations of the incident field, and the multiplicative dependence of the photometric function on albedo. By defining the Signal to Noise Ratio (SNR) of $K-\gamma H$, or a sample mean of $n$ independent and identically distributed measurements of $K-\gamma H$, as the ratio of the squared mean to variance, SNR is proportional to sample size $n$. It also becomes large as the mean photocount becomes large and the standard deviation of albedo becomes small compared to the mean albedo.

\subsection{Maximum Likelihood Estimation}

The likelihood function for an estimate of $\theta$ is defined as $P_{\mathbf{K}}(\mathbf{K} \mid \theta)$ evaluated at the measured values of $\mathbf{K}$, where $P_{\mathbf{K}}(\mathbf{K} \mid \theta)$ is the conditional probability distribution of a data vector $\mathbf{K}$ of independent and identically distributed photocount measurements $K_{1}, K_{2}, K_{3}, \ldots, K_{n}$ obeying Eqs. 4 through 6 given surface slope parameter $\theta$. Measurements of random photocount, in the vector $\mathbf{K}$, then contain information about surface slope $\theta$ through both the mean and variance of the photocount via Eqs $1-6$. The MLE $\widehat{\theta}$ is defined as the surface slope that maximizes the likelihood function with respect to $\theta$ (Rao, 1966; Fisher, 1956). The Cramer-Rao Lower Bound (CRLB) is the minimum mean square error attainable by any unbiased estimate, regardless of the method of estimation. The CRLB $i^{-1}$ is the inverse the Fisher information, also known as the expected information, which is defined as $i=\left\langle l_{1}^{2}\right\rangle$, 
where $l(\mathbf{K} \mid \theta)=\ln P_{\mathbf{K}}(\mathbf{K} \mid \theta)$ is the log-likelihood function, and $l_{j}=\frac{\partial^{j} l(K \mid \theta)}{\partial \theta^{j}}$.

If the sample size $n$ is sufficiently large, or uncertainty is sufficiently small, the MLE $\widehat{\theta}$ is asymptotically unbiased and obeys the Gaussian distribution

$$
P_{\widehat{\theta}}(\widehat{\theta} \mid \theta)=\sqrt{\frac{i}{2 \pi}} \exp \left(-\frac{i}{2}(\widehat{\theta}-\theta)^{2}\right)
$$

with variance $i^{-1}$ equal to the CRLB (Rao, 1966; Kay, 1993), where (Makris, $1995,1996)$

$$
\begin{aligned}
i & =n\left(\frac{1}{\sigma_{K}^{2}}\left(\frac{\partial \bar{K}}{\partial \theta}\right)^{2}+\frac{1}{2}\left(\frac{\partial \ln \left(\sigma_{K}^{2}\right)}{\partial \theta}\right)^{2}\right) \\
& =\frac{n}{\sigma_{K}^{2}}\left(\frac{\partial \bar{K}}{\partial \theta}\right)^{2}\left(1+\frac{1}{2 \sigma_{K}^{2}}\left[1+2 \bar{K} \frac{\sigma_{B o}^{2}}{\overline{B o}^{2}}\right]^{2}\right)
\end{aligned}
$$

given the probability distribution for $K$ described in Eq. 4-6 and Appendix A, Eq. 33. In the deterministic limit $n \rightarrow \infty$, where $K$ is obtained from exhaustive sample averages, $P_{\widehat{\theta}}(\widehat{\theta} \mid \theta)$ becomes the delta function $\delta(\widehat{\theta}-\theta)$.

In photoclinometry, surface slope estimates are obtained from single images, so the sample size is actually $n=1$ and the MLE often will be biased and not attain minimum variance. The necessary sample sizes for the MLE to become effectively unbiased and have a Mean Square Error (MSE) that asymptotically attains the CRLB are derived in Appendix B and appear in Eqs. 39-40. For convenience, we define the necessary minimum sample size, $n_{b}$, to obtain an unbiased MLE by conservatively requiring that the first-order bias $b_{1}$ (Eq. 41) be 10 times smaller than the true value of the parameter,

$$
n_{b}=10 \frac{\left|b_{1}(\widehat{\theta} \mid \theta)\right|}{|\theta|}
$$

Similarly, the necessary minimum sample size, $n_{v}$, for the MSE of an unbiased estimate to attain the CRLB is defined by requiring that the second-order 
variance $\operatorname{var}_{2}$ (Eq. 43) be 10 times smaller than the CRLB,

$$
n_{v}=10 \frac{\left|\operatorname{var}_{2}(\hat{\theta} \mid \theta)\right|}{\operatorname{var}_{1}(\hat{\theta} \mid \theta)}
$$

where $\operatorname{var}_{1}=i^{-1}$ is the CRLB (Eq. 42).

\section{Results and Discussion}

In this section we calculate the exact theoretical biases and errors of photoclinometric surface slope estimates for photometric functions following Lambert's law, Minnaert's law, the lunar-Lambert model, for a typical Martian surface imaging scenario (see Appendix A) using the statistical formulation of Section 2.3 and the Appendices.

To calculate the exact theoretical bias and Root Mean Square Error (RMSE) of a MLE surface slope estimate $\widehat{\theta}$ it is useful to observe that for $\bar{K}=g(\theta)$ and $\theta=g^{-1}(\bar{K})$, it follows that $\widehat{\theta}=g^{-1}(\widehat{\bar{K}})$ by invariance of the MLE (Kay, 1993) where $\widehat{\bar{K}}=K$ is the MLE of the mean photocount $\bar{K}$. The bias and RMSE of $\widehat{\theta}$ are then given by

$$
\begin{aligned}
\operatorname{bias}(\widehat{\theta}) & =\theta-\langle\widehat{\theta}\rangle \\
\operatorname{RMSE}(\widehat{\theta}) & =\sqrt{\operatorname{bias}^{2}(\widehat{\theta})+\operatorname{var}(\widehat{\theta})}
\end{aligned}
$$

where

$$
\begin{aligned}
\langle\widehat{\theta}\rangle & =\int_{0}^{\infty} g^{-1}(K) P_{K}(K \mid \theta) \mathrm{d} K \\
\operatorname{var}(\widehat{\theta}) & =\int_{0}^{\infty}\left(g^{-1}(K)-\langle\widehat{\theta}\rangle\right)^{2} P_{K}(K \mid \theta) \mathrm{d} K
\end{aligned}
$$

for the conditional probability distribution defined in Eqs. 4 through 6. The exact theoretical bias and RMSE are calculated using Eqs. 11-14 for the 
combined effects of all variance terms in Eq. 6 assuming $\sigma_{R}^{2} \approx 6400$ electrons, $\bar{K} \approx O\left(10^{4}\right)$ electrons, $\sigma_{\text {haze }}^{2} \approx 2000$ electrons, and $\sigma_{B o}=0.1 \times \overline{B o}$ as discussed in Appendix A. Results are shown as a function of the incident angle with respect to the zenith direction, $\iota_{z}$, and true surface slope $\theta$ in Figs. 5-7.

Both the bias and RMSE of the surface slope estimate increase significantly in the region where the first derivative of $I$ with respect to $\theta$ goes to zero, and the measurement becomes 'insensitive' to the parameter to be estimated. For the Lambertian photometric function (Fig. 5) the worst errors then occur along the $\iota_{n}=0$ line, a consequence of Lambert's cosine law, as expected from Fig. 2. For the lunar photometric function (Fig. 6), the bias and variance of the estimate are worst along the line $\iota_{z}=\epsilon_{z}$ where the incident and observation directions become collinear, as noted in Fig. 3. Finally, the worst bias and errors for the lunar-Lambert photometric function (Fig. 7) occur along a curve that lies in the region between the $\iota_{n}=0$ and $\iota_{z}=\epsilon_{z}$ curves, depending on the exact weighting between the Lambertian and lunar functions. By using the full likelihood function for the surface slope estimate $\widehat{\theta}$, we find that previous approximations to the biases and errors typically underestimated their true values by as much as $50 \%$, as can be seen by comparing for example the error ranges shown in Fig.A4a of Jankowski and Squyres (1991) to those presented in Fig. 7. The firstorder error term of Jankowski and Squyres (1991), for example, is based on an implicit assumption of additive signal-independent noise, and so is not consistent with the dominant sources of photoclinometric noise, albedo and camera shot noise, which are multiplicative and signal-dependent, and does 
not equal the first-order term expected from estimation theory, the square root of the CRLB (Eq. 42).

\section{[Figure 5, Figure 6, Figure 7]}

Since the bias and RMSE for the lunar-Lambert photometric function may be as large as 10 degrees or more when the incident angle with respect to the zenith direction, $\iota_{z}$ is less than 20 degrees (Fig. 7), obtaining optimal estimates may then necessitate averaging over statistically independent measurements. Here, an optimal estimate is defined as one that is unbiased (or has a bias that is negligible compared to the true value of the parameter), and its RMSE attains the the specified design threshold. Statistically independent samples can be obtained, for example, by measuring surface radiance under different illumination and/or observation conditions, or by estimating surface slopes over larger regions that can be divided into statistically independent and identically distributed sub-regions. When averaging over a spatial region, the correlation area of albedo variability will limit the total number of statistically independent samples available in that region to the ratio of the total area of the region to the correlation area. The number of samples $N$ necessary to attain the design threshold is given by

$$
\sqrt{N}=\frac{\operatorname{RMSE}(\widehat{\theta})}{\text { design threshold }}
$$

By calculating the sample sizes necessary to asymptotically obtain optimal estimates, we find that, while one sample appears to be enough for most illumination conditions, the required number of samples increases significantly in the region of the $\mathrm{d} I / \mathrm{d} \theta=0$ curve. The necessary sample sizes are computed using Eqs. 9-10, 41-43, and shown in Figs. 8-10. In each figure, the white dashed line denotes the curve where $\mathrm{d} I / \mathrm{d} \theta$ goes to zero and more 
than $10^{4}$ samples are typically required to obtain an estimate that asymptotically becomes unbiased and has a RMSE that attains the square root of the CRLB. In Figs. 8(a) and 10(a), a ridge occurs at $\theta=0$, where the denominator vanishes according to our definition for $n_{b}$ (Eq. 9).

[Figure 8, Figure 9, Figure 10]

Figure 11 shows the first-order bias (Eq. 41) and the square root of the CRLB (Eq. 42) for the lunar-Lambert photometric function of Eqs. 2-3 using $L=0.55$. We find that these asymptotic biases and variances differ dramatically from the exact theoretical values, as can be seen by comparing Figs. 11 to 7. This is especially evident in two regimes: (1) at large incidence angles, larger than typically 10 degrees, where the asymptotic biases and errors go to zero, and (2) at small incidence angles, where the asymptotic biases and errors very rapidly approach infinity as $\mathrm{d} I / \mathrm{d} \theta$ goes to zero along the white dashed lines in Fig. 11. In the special case when $n_{v}$ samples are available, the RMSE equals the square root of the CRLB. Even then, the CRLB may still be larger than the design threshold, in which case a total of $N=n_{v} \times n^{\prime}$ samples would be necessary, where $\sqrt{n^{\prime}}=\sqrt{\mathrm{CRLB}} /($ design threshold $)$.

\section{[Figure 11]}

One way of obtaining more independent samples is to tilt the satellite camera to an off-nadir direction. For example, consider the case where the emission direction is at an angle of 20 degrees to the zenith direction, but still lies in the solar plane as defined in Fig. 1 and Section 2.1. The photometric function, the bias and RMSE (Eqs. 11-14) of the MLE, and the necessary sample size conditions for this case are shown in Figs. 12-14.

Comparing Figs. 7 and 10 to Figs. 13 and 14, respectively, we find that 
rotating the camera significantly affects the bias and RMSE, as well as the sample size necessary to obtain an optimal surface slope estimate for given values of $\iota_{z}$ and $\theta$. This example then suggests that carefully designed offnadir viewing may provide an opportunity for reducing surface slope biases and errors when combined with nadir images.

Depending on the exact experimental conditions, it may be possible to specify a general strategy where an optimal estimate can be obtained from a single sample. For example, for the two cases of the lunar-Lambert function presented here (Figs. 4 and 12), requiring $\left|\iota_{z}\right|>30^{\circ}$, and $\left|\iota_{z}\right|>\left|\epsilon_{z}\right|+20^{\circ}$ will allow optimal estimates to be obtained for most values of $\theta$. These two conditions are less stringent than the $\left|\iota_{z}\right| \approx 60^{\circ}-75^{\circ}$, which is typically specified as the optimal regime for photoclinometry (e.g. Davis and McEwen, 1984; Jankowski and Squyres, 1991), where the upper limit typically stems from the need to avoid shadows which are not amenable to investigation. The results presented here (Figs. 5-7 and 13) suggest that photoclinometry may work equally well even at shallower incidence angles.

\section{[Figure 12, Figure 13, Figure 14]}

\subsection{Comparison of the Different Sources of Noise or Uncertainty}

Here, we examine the biases and errors due to each source of noise or uncertainty described in Section 3 acting in the absence of the others. Specifically, biases and errors are calculated with Eqs. 11-14 by replacing Eq. 6 with only the variance term for either (i) read noise, (ii) shot noise, (iii) atmospheric haze, or (iv) albedo variability for each respective case.

As expected, the biases and errors for each noise source increase significantly in the region where the reflectance function has weak dependence on 
surface slope, as shown in Figures 15 and 16. The total bias and RMSE for all these error sources has been shown in Fig. 7. Biases often dominate the RMSEs.

We find that albedo variability is typically the dominant source of biases and errors, on the order of $10-20^{\circ}$ or more at small incidence angles $\left(\iota_{n}\right.$ smaller than roughly ten degrees), as can be seen from Fig. 15(d). Camera shot and read noise are the next most important noise sources, leading to biases and errors on the order of $5^{\circ}$ (Fig. 15(a-b)). Finally, haze appears to be the least significant source of noise, resulting in biases and errors that are typically on the order of a $1-2^{\circ}$, or approximately an order of magnitude smaller than those due to albedo variability, as can be seen from Fig. 15(c). This last result is in agreement with previous literature (Kirk et al., 2003b), where errors due to haze have typically been found to comprise less than $20 \%$ of the total error when accurately modeled. Note however that haze effects may increase significantly in magnitude during dust storms (Cantor et al., 2001).

[Figure 15, Figure 16]

Since the ratio of the albedo to the shot noise contribution of the photocount variance from Eq. 6 is $\bar{K} \frac{\sigma_{B O}^{2}}{\overline{B o}}$, we expect that albedo noise should dominate the variance of the photocount $K$ if the standard deviation of albedo uncertainty is larger than $\frac{1}{\sqrt{K}}$ times the mean albedo, or roughly $1 \%$ of the mean albedo for the mean photocount used here of $\bar{K} \approx O\left(10^{4}\right)$, given sufficiently low read and haze noise contributions. This is indeed found to be the case in Figs. 15-16 where $\sigma_{B o}=0.1 \times \overline{B o}$, for our typical Martian scenario. For much lower albedo uncertainty of $\sigma_{B o}=0.005 \times \overline{B o}$, as reported for Miranda (Hillier et al., 1989), the total bias and error are instead domi- 
nated by shot noise, which can be seen by comparing the black dashed lines to the gray dash-dotted lines in Fig. 17 which shows a cut through Figs. 7 and 15-16 along the line $\iota_{z}=12^{\circ}$.

[Figure 17]

\section{Conclusions}

Both theoretically exact and asymptotic biases and errors inherent in photoclinometric estimation of planetary surface orientation from ChargeCoupled Device (CCD) measurements are calculated using an approach developed from classical estimation theory. The approach can be used to determine the accuracy of topographic reconstructions and aid in experimental design.

The likelihood function governing statistical fluctuations of a photoclinometric slope estimate is derived, including uncertainty due to camera shot noise, camera read noise, small-scale albedo fluctuations and atmospheric haze. The derivation incorporates common photometric models of planetary surface reflectance and the known probability distributions of CCD measurements of natural light. From this, bounds on the minimum mean square error of any unbiased estimate of photoclinometric surface slope are derived, as are necessary conditions to attain these bounds and constrain errors within desired design thresholds. Approximate asymptotic biases and errors for low uncertainty (1) are formulated in terms of the likelihood function to insure proper convergence with decreasing uncertainty, and (2) typically differ dramatically from the exact ones, making them applicable only when a large number of independent samples is available. Biases and errors are shown to 
typically become much larger than surface slopes for illuminations and observations where planetary reflectance is weakly dependent on surface slope, near inflection points of the photometric function.

The approach developed here provides a unified method for quantitatively comparing the biases and errors from different sources of uncertainty in a photoclinometric estimate. Albedo variability, for example, is shown to typically dominate estimate biases and errors when the standard deviation of

albedo uncertainty is larger than approximately $\frac{1}{\sqrt{K}}$ times the mean albedo in the imaged region, for CCD photocount $K$, while other error sources such as shot noise may become dominant for very low albedo uncertainty.

\section{A. Statistics of CCD Measurements of Surface Reflectance}

We show that a CCD photocount measurement, $K$, of planetary surface reflectance from a natural light source approximately follow a Gaussian distribution. The derivation incorporates surface albedo variability, as well as CCD camera read and shot noise, and atmospheric haze noise. The number of photoevents $K$ recorded by CCD cameras is directly proportional to incident intensity (Janesick, 2001). The averaged intensity incident on a photosurface of area $A$ in the time interval $\left(t_{0}, t_{0}+\tau\right)$ is a random variable

$$
W \mid B o=\frac{1}{\tau A} \iiint_{t_{0}}^{t_{0}+\tau} \mathscr{I}(x, y ; t \mid B o) \mathrm{d} t \mathrm{~d} x \mathrm{~d} y
$$

where $\mathscr{I}(x, y ; t \mid B o)$ is the random instantaneous intensity at time $t$ and location $(x, y)$ on the photosurface given albedo $B o$. For satellite imaging of a planetary surface under the illumination-observation scenario described in Fig. 1, the expected value of $\mathscr{I}(x, y ; t \mid B o)$ is proportional to the surface 
reflectance function $I\left(\mu_{n}, \mu_{0 n}, \alpha\right)$ times the incident solar flux $\mathscr{I}_{0}$ (McCluney, 1994, chap. 1), where the latter is assumed to be a known constant, plus the mean intensity from atmospheric haze $H$. The surface reflectance function can be expressed as $I\left(\mu_{n}, \mu_{0 n}, \alpha\right)=B o f(\theta)$ (Eq. 3), where $B o$ is the random surface albedo and $\theta$ is the unknown planetary surface slope. Atmospheric haze is described by a CCGR field that is independent and additive to the CCGR field from the surface, so that the field variances, or equivalently the mean instantaneous intensities of each add.

The probability distribution of $W \mid B o$ for polarized thermal light is given by the Gamma distribution (Goodman, 1985, chap. 9),

$$
P_{W \mid B o}(W \mid B o)= \begin{cases}\left(\frac{\zeta}{\bar{W}_{B o}}\right)^{\zeta} \frac{W^{\zeta-1} \exp \left(-\zeta \frac{W}{\bar{W}_{B o}}\right)}{\Gamma(\zeta)} & \text { for } W \geq 0 \\ 0 & \text { for } W<0\end{cases}
$$

where $\bar{W}_{B o} \equiv\langle W \mid B o\rangle=\int W P_{W \mid B o}(W \mid B o) \mathrm{d} W=\mathscr{I}_{0}[B o f(\theta)+H]$, and $H$ is the expected intensity of atmospheric haze which is assumed to be a known constant (e.g. Beyer et al., 2003). The variable $\zeta$ is the number of coherence cells in the intensity average (Goodman, 1985, chap. 6) which is equal to the squared-mean-to-variance ratio, or Signal to Noise Ratio (SNR) of $W \mid B o$, defined as $\langle W \mid B o\rangle^{2} /\left(\left\langle W \mid B o^{2}\right\rangle-\langle W \mid B o\rangle^{2}\right)$. For example, $\zeta$ equals the time-bandwidth product of the received field if $W \mid B o$ is obtained from a finite-time average (Makris, 1995). Additionally, $\zeta$ can be interpreted as the number of stationary speckles averaged over a finite spatial aperture in the image plane or the number of stationary multi-look images averaged for a particular scene (Arsenault and April, 1976; Makris, 1995).

The probability of observing $K$ photoevents follows the conditional Pois- 
son distribution (Goodman, 1985, chap. 9)

$$
P_{K \mid W, B o}(K \mid W, B o)=\frac{\left(\bar{K}_{W, B o}\right)^{K}}{K !} \mathrm{e}^{-\bar{K}_{W, B o}}
$$

where $\bar{K}_{W, B o}=\beta \tau A W$. The proportionality constant $\beta$ is given by $\beta=\frac{\eta}{h \bar{\nu}}$, where $h$ is Planck's constant $\left(6.626 \times 10^{-34}\right.$ Joule-s $), \bar{\nu}$ is the mean optical frequency of radiation, and $\eta$ is the quantum efficiency that represents the average number of photoevents produced by each incident photon $(0 \leq \eta \leq$ 1). From Eqs. 17 and 18, the probability of observing $K$ photoevents then follows the negative binomial distribution

$$
\begin{aligned}
P_{K \mid B o}(K \mid B o) & =\int_{-\infty}^{\infty} P_{K \mid W, B o}(K \mid W, B o) P_{W \mid B o}(W \mid B o) \mathrm{d} W \\
& =\frac{\Gamma(K+\zeta)}{\Gamma(K+1) \Gamma(\zeta)}\left[1+\frac{\zeta}{\bar{K}_{B o}}\right]^{-K}\left[1+\frac{\bar{K}_{B o}}{\zeta}\right]^{-\zeta}
\end{aligned}
$$

where $\bar{K}_{B o}=\beta \tau A \bar{W}_{B o} \equiv \gamma[\operatorname{Bof}(\theta)+H]$, and we have defined for convenience the proportionality constant $\gamma$

$$
\gamma \equiv \beta \tau A \mathscr{I}_{0}=\frac{\eta}{h \bar{\nu}} \tau A \mathscr{I}_{0}
$$

For $\zeta \gg 1$, the Gamma distribution of Eq. 17 approximates a delta function (Mandel, 1959), $P_{W \mid B o}(W \mid B o)=\delta\left(W-\bar{W}_{B o}\right)$, so that the negative binomial distribution for $K$ (Eq. 19) approaches a Poisson distribution. To show this, let $q=\bar{K}_{B o} / \zeta$. The first cumulant of the negative binomial distribution for $K$ conditional on $B o$ is given by $\lambda_{1}\left(K \mid B_{o}\right)=\zeta q$, and the rest by the recursion equation

$$
\lambda_{j+1}(K \mid B o)=q(q+1) \frac{\mathrm{d} \lambda_{j}(K \mid B o)}{\mathrm{d} q},
$$

so that

$$
\lambda_{n}(K \mid B o)=\zeta\left(q+\sum_{j=2}^{n} a_{j} q^{j}\right) \quad \text { for } n \geq 2
$$


where the $a_{j}$ are constants. For thermal light at optical frequencies, and for the common integration times of CCDs, $\zeta$ is very large, usually in the order of $10^{10}$, while maximum values for $\bar{K}_{B o}$ are typically much smaller, around $10^{4}$. For $\zeta \gg \bar{K}_{B o}, q$ tends to 0 and $\lambda_{n}(K \mid B o) \approx \zeta q$ for all $n$, so that the cumulants of $K \mid B o$ become equal to those of a Poisson distributed random variable with mean $\zeta q=\bar{K}_{B o}$, which is random since $B o$ is a random variable.

The total probability distribution for $K$ is also approximately Gaussian by virtue of the central limit theorem. To show this, we first calculate the cumulants $\kappa_{n}$ of the photocount $K$ using the law of total cumulance (Brillinger, 1969),

$$
\kappa_{n}(K) \equiv \kappa_{1}\left(K_{1}, \ldots, K_{n}\right)=\sum_{\pi} \kappa_{1}\left(\lambda_{1}\left(K_{\pi 1} \mid B o\right), \ldots, \lambda_{1}\left(K_{\pi j} \mid B o\right)\right)
$$

where the summation is defined over all possible partitions $\pi$ of the set $\{1, \ldots, n\}$ of indices. For each partition $\pi$, sub-blocks are denoted by $\pi 1, \ldots, \pi j$, so that for example, if $n=3$ and $\pi=\{2$ indices, 1 index $\}$, the sub-blocks are $\pi_{1}=\{[1,2], 3\}, \pi_{2}=\{[1,3], 2\}$, and $\pi_{3}=\{[2,3], 1\}$. We then define $K_{1}=K_{2}=\ldots=K_{n}=K$. Equation 23 reduces to the well known laws of iterated expectations and total variance for $n=1,2$ (Bertsekas and Tsitsiklis, 2008). The cumulants for $K$ are given by,

$$
\begin{aligned}
\kappa_{1}(K) & =\left\langle\bar{K}_{B o}\right\rangle \\
\kappa_{2}(K) & =\left\langle\bar{K}_{B o}\right\rangle+\operatorname{var}\left(\bar{K}_{B o}\right), \\
\vdots & \\
\kappa_{n}(K) & =\left\langle\bar{K}_{B o}\right\rangle+b_{n} \operatorname{var}\left(\bar{K}_{B o}\right)
\end{aligned}
$$

where the $b_{n}$ are constants and we have made use of $\kappa_{j}\left(\bar{K}_{B o}\right)=0$ for all 
$j \geq 3$, since $B o$ has been assumed to follow a Gaussian distribution with mean $\overline{B o}$ and variance $\sigma_{B o}^{2}$.

Defining a new random variable

$$
U=\frac{(K-\bar{K})}{\sqrt{\bar{K}}\left(1+\bar{K} \sigma_{B o}^{2} / \overline{B o}^{2}\right)^{1 / 2}}
$$

where $\bar{K}=\left\langle\bar{K}_{B o}\right\rangle=\gamma[\overline{B o} f(\theta)+H]$, the cumulant generating function of $U$ is given by

$$
\begin{aligned}
g_{U}(\phi) & =\ln \left\langle\mathrm{e}^{\phi U}\right\rangle \\
& =-\phi \frac{\sqrt{\bar{K}}}{\left(1+\bar{K} \sigma_{B o}^{2} / \overline{B o}^{2}\right)^{1 / 2}}+g_{K}\left(\frac{\phi}{\sqrt{\bar{K}}\left(1+\bar{K} \sigma_{B o}^{2} / \overline{B o}^{2}\right)^{1 / 2}}\right),
\end{aligned}
$$

where $g_{K}(\phi)$ is the cumulant generating function of $K$. The cumulants of $U$ are then given by

$$
u_{j}=\left.\frac{\mathrm{d}^{j} g_{U}(\phi)}{\mathrm{d} \phi^{j}}\right|_{\phi=0}=\frac{1}{\left[\sqrt{\bar{K}}\left(1+\bar{K} \sigma_{B o}^{2} / \overline{B o}^{2}\right)^{1 / 2}\right]^{j}}\left(-\left.\bar{K}^{j} \frac{\mathrm{d}^{j} \phi}{\mathrm{d} \phi^{j}}\right|_{\phi=0}+\kappa_{j}(K)\right),
$$

so that

$$
\begin{aligned}
u_{1} & =0, \\
u_{2} & =1, \\
\vdots & \bar{K}\left(1+c_{n} \bar{K} \sigma_{B o}^{2} / \overline{B o}^{2}\right) \\
u_{n} & =\frac{\left.\bar{K}\left(1+\bar{K} \sigma_{B o}^{2} / \overline{B o}^{2}\right)^{1 / 2}\right]^{n}}{[\sqrt{\bar{K}(10 r} n \geq 2,}
\end{aligned}
$$

where the $c_{n}$ are constants. For very large values of $\bar{K}, u_{n}$ approaches 0 for $n>2$, so that $U$ and consequently $K$ become Gaussian random variables.

The discrete probability density for $K$ is then well approximated by the continuous Gaussian probability distribution,

$$
P_{K}(K \mid \theta)=\frac{1}{\sqrt{2 \pi} \sigma_{K}(\theta)} \exp \left(-\frac{1}{2}\left[\frac{K-\bar{K}(\theta)}{\sigma_{K}(\theta)}\right]^{2}\right)
$$


where

$$
\begin{aligned}
\bar{K} & =\gamma[\overline{B o} f(\theta)+H] \\
\sigma_{K}^{2} & =\bar{K}+\bar{K}^{2} \sigma_{B o}^{2} \overline{B o}^{2}+\sigma_{R}^{2}
\end{aligned}
$$

are the mean and variance of $K$ respectively, $\sigma_{R}^{2}$ is an additive signal-independent variance term due to CCD camera read noise, and we define $\sigma_{\text {haze }}^{2} \equiv \gamma H$ as the noise term due to the known atmospheric haze expected intensity $H$.

In order to determine appropriate noise levels and the scaling factor $\gamma$, we consider as an example the HiRISE camera of the latest Mars Reconnaissance Orbiter (MRO) mission (Bergstrom et al., 2004; McEwen et al., 2003), where Bergstrom et al. (2004) specify read noise $\sigma_{R}$ to be roughly 80 electrons r.m.s., and imply in Fig. 12 a $\gamma$ ranging from roughly 20000 electrons for the bluegreen and NIR bands to 70000 electrons for the red (pan) band. The mean photocount, $\bar{K}$, is then on the order of $10^{4}$ electrons.

A typical value for the contribution of atmospheric haze to the total measured signal in images of Mars may be inferred from Table I of Bridges and Herkenhoff (2002). Accounting for the gain of Mariner's camera (Dunne, 1970), we find that the atmospheric haze component, $\sigma_{\text {haze }}^{2}$, is typically on the order of 2000 electrons, or roughly $10 \%$ of the mean signal expected using the blue-green HiRISE band. For albedo variability, the mean $\overline{B o}$ is normalized to one and the standard deviation $\sigma_{B o}$ is specified as $10 \%$ of the mean following calculations presented by Bell et al. (2008) for typical Martian surfaces. Albedo variability then results in a variance that is on the order of $10^{6}$ electrons.

The integration time or shutter speed of the HiRISE camera is $\tau \geq 76$ $\mu$ sec and the optical bandwidth is $\nu_{B} \approx 10^{14} \mathrm{~Hz}$ or greater, depending on 
the exact band used. The value of $\zeta$ is then in the order of $10^{10}$, while $\bar{K}$ is in the order of $10^{4}$, so that the negative binomial distribution of Eq. 19 is well approximated by the Gaussian distribution of Eq. 33. In this paper, we consider CCD measurements in the blue-green and/or the NIR band.

\section{B. Necessary Sample Sizes for an Unbiased, Minimum Variance Estimate, and Asymptotic Expansions of Bias and Variance}

Given the likelihood function $P_{\mathbf{K}}(\mathbf{K} \mid \theta)$ for $\theta$ given the measurements $K_{1}, K_{2}, K_{3}, \ldots, K_{n}$, the moments of the MLE estimate $\widehat{\theta}$ can be expressed as series of inverse powers of the sample size $n$ (Naftali and Makris, 2001), provided that the required derivatives in an expansion of the likelihood function exist (Shenton and Bowman, 1977). The MLE variance is then expressed as

$$
\operatorname{var}(\widehat{\theta}, n)=\operatorname{var}_{1}(\hat{\theta} \mid \theta, n)+\operatorname{var}_{2}(\hat{\theta} \mid \theta, n)+\text { Higher Order terms }
$$

where $\operatorname{var}_{j}(\widehat{\theta} \mid \theta, n)=\operatorname{var}_{j}(\widehat{\theta} \mid \theta, 1) / n^{j}$, so that

$$
\operatorname{var}(\widehat{\theta}, n)=\frac{\operatorname{var}_{1}(\widehat{\theta} \mid \theta, 1)}{n}+\frac{\operatorname{var}_{2}(\widehat{\theta} \mid \theta, 1)}{n^{2}}+O\left(n^{-3}\right)
$$

where $O\left(n^{-3}\right)$ represents integer powers $n^{-3}$ and higher. The first term on

the right hand side, $\operatorname{var}_{1}(\widehat{\theta} \mid \theta, 1) / n$ is the CRLB, the asymptotic value of the variance when sample size $n$ becomes large or uncertainty becomes small. Similarly, the MLE bias can be expressed as

$$
\operatorname{bias}(\widehat{\theta}, n)=\frac{b_{1}(\hat{\theta} \mid \theta, 1)}{n}+\frac{b_{2}(\hat{\theta} \mid \theta, 1)}{n^{2}}+O\left(n^{-3}\right)
$$

To simplify notation, we let $\operatorname{var}_{j}(\widehat{\theta} \mid \theta, 1) \equiv \operatorname{var}_{j}(\widehat{\theta} \mid \theta)$ and $b_{j}(\widehat{\theta} \mid \theta, 1) \equiv b_{j}(\widehat{\theta} \mid \theta)$. 
The value of $n$ necessary for the MLE variance to asymptotically attain the CRLB is found by requiring the second-order variance to be negligible compared to the first-order variance, so that

$$
n \gg \frac{\left|\operatorname{var}_{2}(\widehat{\theta} \mid \theta)\right|}{\operatorname{var}_{1}(\hat{\theta} \mid \theta)}
$$

Similarly, the necessary value of $n$ for $\hat{\theta}$ to become asymptotically unbiased is found by requiring the first-order bias to be negligible compared to the true value of the parameter

$$
n \gg \frac{\left|b_{1}(\hat{\theta} \mid \theta)\right|}{|\theta|}
$$

Only for values of $n$ satisfying these conditions is it possible for the variance to be in the asymptotic regime where it is unbiased and continuously attains the CRLB (Naftali and Makris, 2001; A. Thode et al., 2002; Zanolin et al., 2004).

For the statistical model of Eq. 33

$$
\begin{aligned}
& b_{1}(\widehat{\theta} \mid \theta)=-\frac{1}{2}\left(i^{-2}\right)\left[v_{1,2}+\frac{1}{2} \nu_{1} \nu_{2}+v_{1,1} \nu_{1}\right] \\
& \operatorname{var}_{1}(\widehat{\theta} \mid \theta)= i^{-1}, \\
& \operatorname{var}_{2}(\widehat{\theta} \mid \theta)=\left(i^{-3}\right)\left[2 \nu_{1}^{4}-5 v_{1,2} \nu_{1}+6 v_{1,1} \nu_{1}^{2}-\frac{1}{2} \nu_{1} \nu_{3}\right. \\
&\left.-v_{1,1} \nu_{2}-v_{1,3}-\nu_{1}^{2} \nu_{2}\right] \\
&+\left(i^{-4}\right)\left[\frac{7}{2} v_{1,2}^{2}+\frac{7}{2} v_{1,1} \nu_{1}^{2} \nu_{2}+7 v_{1,1} v_{1,2} \nu_{1}\right. \\
&+\frac{7}{8} \nu_{1}^{2} \nu_{2}^{2}+\frac{7}{2} v_{1,2} \nu_{1} \nu_{2}-\frac{11}{2} v_{1,1}^{2} \nu_{1}^{2} \\
&\left.-6 v_{1,1} \nu_{1}^{4}-\nu_{1}^{6}\right]
\end{aligned}
$$

where

$$
v_{a, b}=\frac{1}{\sigma_{K}^{2}} \frac{\partial^{a} \bar{K}}{\partial \theta^{a}} \frac{\partial^{b} \bar{K}}{\partial \theta^{b}}
$$




$$
\nu_{c}=\frac{1}{\sigma_{K}^{2}} \frac{\partial^{c} \sigma_{K}^{2}}{\partial \theta^{c}}
$$

and $i$ is the Fisher information given by Eq. 8 . 


\section{References}

Arsenault, H. H., April, G. 1976. Properties of speckle integrated with a finite aperture and logarithmically transformed. J. Opt. Soc. Am. 66, 1160-1163.

Barndorff-Nielsen, O. E., Cox, D. R. 1994. Inference and Asymptotics. Chapman and Hall, London.

Bell, J. F. III, Rice, M. S., Johnson, J. R., Hare, T.M. 2008. Surface albedo observations at Gusev Crater and Meridiani Planum, Mars. J. Geophys. Res. 113, E06S18.

Bergstrom, J. W., Delamere, W. A., McEwen, A. 2004. MRO High Resolution Imaging Science Experiment (HiRISE): Instrument Test, Calibration and Operating Constraints. 55th International Astronautic Federation Congress, IAC-04-Q.3.b.02 unpublished.

Bertsekas, D. P., Tsitsiklis, J. N. 2008. Introduction to Probability, 2nd edition. Athena Scientific, Belmont, MA.

Beyer, R. A., McEwen, A. S., Kirk, R. L. 2003. Meter-Scale Slopes of Candidate MER Landing Sites from Point Photoclinometry. J. Geophys. Res. 108,8085 .

Bridges, N. T., Herkenhoff, K. E. 2002. Topography and Geologic Characteristics of Aeolian Grooves in the South Polar Layered Deposits of Mars. Icarus 156, 387-398.

Brillinger, D. 1969. The calculation of cumulants via conditioning. Annals of the Institute of Statistical Mathematics, Vol. 21, pp. 215-218. 
Cantor, B. A., James, P. B., Caplinger, M., Wolff, M. J. Martian dust storms: 1999 Mars Orbiter Camera observations. J. Geophys. Res. 106, 2365323687.

Davis, P. A., Soderblom, L. A. 1984. Modeling Crater Topography and Albedo From Monoscopic Viking Orbiter Images, 1. Methodology. J. Geophys. Res. 89, 9449-9457.

Davis, P. A., McEwen, A. S. 1984. Photoclinometry: Analysis of Inherent Errors and Implications for Topographic Measurements. Planet. Sci. IV, 194-195.

Dunne, J. A. 1970. Mariner 1969 Television Image Processing. Pattern Recognition 2, 261-266.

Efford, N. D. 1991. Sources of Error in the Photoclinometric Determination of Planetary Topography: A Reappraisal. Earth Moon Planets 54, 19-58.

Fisher, R. A. 1956. Statistical Methods and Scientific Inference. Hafner, New York.

Goodman, J. W. 1985. Statistical Optics. Wiley, New York.

Hillier, J., Helfenstein, P., Veverka, J. 1989. Miranda: Color and Albedo Variations from Voyager Photometry. Icarus 82, 314-335.

Howard, A. D. 1982. Photoclinometric Determination of the Topography of the Martian North Polar Cap. Icarus 50, 245-258.

Janesick, J. R. 2001. Scientific Charge-Coupled Devices. SPIE Press. 
Jankowski, D. G., Squyres, S. W. 1991. Sources of Error in Planetary Photoclinometry. J. Geophys. Res. 196, 20907-20922.

Kay, S. M. 1993. Fundamentals of Statistical Signal Processing: Estimation Theory. Prentice Hall, New Jersey.

Kirk, R. L., Thompson, K. T., Lee, E. M. 2001. Photometry of the Martian Atmosphere: An Improved Practical Model for Cartography and Photoclinometry. Lunar Planet. Sci. XXXII.

Kirk, R. L., Barrett, J. M., Soderblom, L. A. 2003. Photoclinometry made simple...? Advances in Planetary Mapping, Houston.

Kirk, R. L., Howington-Kraus, E., Redding, B., Galuszka, D., Hare, T. M., Archinal, B. A., Soderblom, L. A., Barrett, J. M. 2003. High-Resolution Topomapping of Candidate MER Landing Sites with Mars Orbiter Camera Narrow-Angle Images. J. Geophys. Res. 108, 8088.

Makris, N. C. 1995. A foundation for logarithmic measures of fluctuating intensity in pattern recognition. Optics Letters 20, 2012-2014.

Makris, N. C. 1996. The effect of saturated transmission scintillation on ocean-acoustic intensity measurements. J. Acoust. Soc. Am. 100, 769-783.

Malin, M. C., Danielson, G. E., Ingersoll, A. P., Masursky, H., Veverka, J., Ravine, M. A., Soulanille, T. A. 1992. Mars Observer Camera. J. Geophys. Res. 97, 7699-7718.

Malin, M. C., Edgett, K. S. 2001. Mars Global Surveyor Mars Orbiter Cam- 
era: Interplanetary cruise through primary mission. J. Geophys. Res. 106, 23429-23570.

Mandel, L. 1959. Fluctuations of photon beams: The distribution of photoelectrons. Proc. Phys. Soc. 74, 233-243.

McCluney, R. 1994. Introduction to Radiometry and Photometry. Artech House, Massachusetts.

McCullagh, P. 1987. Tensor Methods in Statistics (Monographs on Statistics and Applied Probability). Chapman and Hall, London.

McEwen, A. S. 1986. Exogenic and endogenic albedo and color patterns on Europa. J. Geophys. Res. 91, 8077-8097.

McEwen, A. S. 1991. Photometric Functions for Photoclinometry and Other Applications. Icarus 92, 298-311.

McEwen, A., 14 colleagues 2003. MRO's High Resolution Imaging Science Experiment (HiRISE): Science Expectations. 6th International Conference on Mars, Pasadena, California.

Naftali, E., Makris, N. C. 2001. Necessary conditions for a maximum likelihood estimate to become asymptotically unbiased and attain the CramerRao Lower Bound. Part I. General approach with an application to timedelay and Doppler shift estimation. J. Acoust. Soc. Am. 110, 1917-1930.

Ranson, K. J., Irons, J. R., Daughtry, C. S. T. 1991. Surface Albedo from Bidirectional Reflectance. Remote Sens. Environ. 35, 201-211. 
Rao, C. R. 1966. Linear Statistical Inference and its Applications. Wiley, New York.

Schenk, P. 2005. Landing Site Characteristics for Europa 1: Topography. Lunar Planet. Sci. XXXVI.

Shenton, L. R., Bowman, K. O. 1977. Maximum Likelihood Estimation in Small Samples. Griffin's, New York.

Thode, A., Zanolin, M., Naftali, E., Ingram, I., Ratilal, P., Makris, N. C. 2002. Necessary conditions for a maximum likelihood estimate to become asymptotically unbiased and attain the Cramer-Rao Lower Bound. Part II. Range and depth localization of a sound source in an ocean waveguide. J. Acoust. Soc. Am. 112, 1890-1910.

Zanolin M., Ingram, I., Thode, A., Makris, N. C. 2004. Asymptotic Accuracy of Geoacoustic Inversions. J. Acoust. Soc. Am. 116, 2031-2042. 


\section{Figure Captions}

FIG. 1: Resolved surface element with slope $\theta$ to flat topography. The slope, or tilt $\theta$ is the angle suspended between the $z$-axis and the surface normal, measured counter-clockwise. All other angles are measured counter-clockwise from the $z$-axis or the surface normal direction, as indicated by subscript $z$ or $n$ respectively. The true incident angle is equal to the angle between the $z$-axis and the incident direction, $\iota_{z}$, minus surface slope, $\theta$, or $\iota_{n}=$ $\iota_{z}-\theta$. Similarly, the true emission angle is equal to the angle between the $z$-axis and the emission direction, $\epsilon_{z}$, minus surface slope, $\theta$, or $\epsilon_{n}=\epsilon_{z}-\theta$. Specular reflection occurs when $\epsilon_{n}=-\iota_{n}$. Given known angles $\iota_{z}$ and $\epsilon_{z}$,

photoclinometry can be used to obtain an estimate of the unknown surface slope $\theta$.

FIG. 2: Lambertian photometric function given constant albedo, Eq. 3 using $L=0$. (a) 3D representation of the value of Eq. 3 for $L=0$ as a function of surface slope $\theta$, which is the parameter to be estimated, and incident angle with respect to flat topography $\iota_{z}$. The emission angle $\epsilon_{z}$ is assumed to be zero so that the satellite is nadir-looking. The black lines correspond to lines of constant true incident angle, $\iota_{n}=\iota_{z}-\theta$. The regions beyond the $\left|\iota_{n}\right|=90^{\circ}$ lines correspond to incidence on the 'back' of the surface patch, so that nothing is reflected towards the receiver and $I=0$. Superimposed on the plot is the curve along which the derivative of $I$ with respect to $\theta$ is zero (white dashed line). Also shown is the line that corresponds to specular reflection, $\epsilon_{n}=-\iota_{n}$ (white dot-dashed line). The plot can also be interpreted 
as a sheared and rotated version of the plot of $I$ versus true incident and emission angle, $\iota_{n}$ and $\epsilon_{n}$ respectively. (b) Three cuts along constant values of incident angle to flat topography, $\iota_{z}$, for the same photometric function. Each curve is obtained by cutting along the corresponding white dotted line in Fig. 2(a) from right to left.

FIG. 3: The same as Fig. 2 for the lunar photometric function given constant albedo, Eq. 3 using $L=1$.

FIG. 4: The same as Fig. 2 for the lunar-Lambert photometric function given constant albedo, Eq. 3 using $L=0.55$, which is a typical value when modeling the reflectance of Martian terrain.

FIG. 5: Absolute value of the bias and Root Mean Square Error (RMSE) (Eqs. 11-14) of the Maximum Likelihood Estimate (MLE) of surface slope for the Lambertian photometric function of Fig. 2 given typical values for the different sources of noise: (i) CCD camera read noise, $\sigma_{R}^{2} \approx 6400$ electrons, (ii) CCD camera shot noise, $\bar{K} \approx O\left(10^{4}\right)$ electrons, (iii) atmospheric haze, $\sigma_{\text {haze }}^{2} \approx 2000$ electrons, and (iv) albedo variability, $\sigma_{B o}=0.1 \times \overline{B o}$ (see Appendix A). (a) Bias as a function of incident angle with respect to flat topography $\iota_{z}$, and true surface slope $\theta$. (b) RMSE as a function of $\iota_{z}$ and $\theta$.

FIG. 6: The same as Fig. 5 for the lunar photometric function of Fig. 3. 
FIG. 7: The same as Fig. 5 for the lunar-Lambert photometric function of Fig. 4.

FIG. 8: Necessary sample sizes to obtain an unbiased estimate of planetary surface slope and for an unbiased estimate to attain the minimum possible RMSE. The planetary surface reflectance is assumed to follow the Lambertian photometric function of Fig. 2. (a) $10 \log _{10}$ of the necessary sample size for an unbiased estimate $\widehat{\theta}$ as a function of incidence angle to flat topography $\iota_{z}$, and true surface slope $\theta$ computed using Eqs. 9 and 41. (b) $10 \log _{10}$ of the necessary sample size for an unbiased estimate to attain the minimum possible RMSE as a function of $\iota_{z}$ and $\theta$ computed using Eqs. 10 and 42-43. The white dashed line indicates the curve along which the derivative of the photometric function with respect to the estimated parameter $\theta$ is zero and the necessary sample sizes approach infinity.

FIG. 9: The same as Fig. 8 for a planetary surface that can be modeled using the lunar photometric function of Fig. 3.

FIG. 10: The same as Fig. 8 for a planetary surface that can be modeled using the lunar-Lambert photometric function of Fig. 4 , where $L=0.55$.

FIG. 11: Absolute value of the first-order bias and the square root of the CRLB, Eqs. 41 and 42, respectively, of the Maximum Likelihood Estimate (MLE) of surface slope for the lunar-Lambert photometric function. (a) 
First-order bias as a function of incident angle with respect to flat topography $\iota_{z}$, and true surface slope $\theta$. (b) Square root of the CRLB as a function of $\iota_{z}$ and $\theta$. The white dashed line indicates the curve along which the derivative of the photometric function with respect to the estimated parameter $\theta$ is zero and the asymptotic biases and errors approach infinity.

FIG. 12: The same as Fig. 4 for an emission angle $\epsilon_{z}=20$ degrees. Again, the regions beyond the $\left|\iota_{n}\right|=90^{\circ}$ lines correspond to incidence on the 'back' of the surface patch, so that nothing is reflected towards the receiver and $I=0$. Note the axes are shifted compared to Fig. 4 to ensure the emission direction never lies behind the surface patch.

FIG. 13: The same as Fig. 7 for an emission angle $\epsilon_{z}=20$ degrees.

FIG. 14: The same as Fig. 10 for an emission angle $\epsilon_{z}=20$ degrees.

FIG. 15: Absolute value of the bias (Eqs. 11 and 13) of the MLE of surface slope for the lunar-Lambert photometric function, given typical values for the different sources of noise: (i) CCD camera read noise, $\sigma_{R}^{2} \approx 6400$ electrons, (ii) CCD camera shot noise, $\bar{K} \approx O\left(10^{4}\right)$ electrons, (iii) atmospheric haze, $\sigma_{\text {haze }}^{2} \approx 2000$ electrons, and (iv) albedo variability, $\sigma_{B o}=0.1 \times \overline{B o}$ (see Appendix A). The emission angle is again assumed to be zero, and $L=0.55$. The total bias has been shown in Fig. 7(a). 
FIG. 16: RMSE (Eqs.12 and 13-14) of the MLE of surface slope for the lunarLambert photometric function, given typical values for the different sources of noise: (i) CCD camera read noise, $\sigma_{R}^{2} \approx 6400$ electrons, (ii) CCD camera shot noise, $\bar{K} \approx O\left(10^{4}\right)$ electrons, (iii) atmospheric haze, $\sigma_{\text {haze }}^{2} \approx 2000$ electrons, and (iv) albedo variability, $\sigma_{B o}=0.1 \times \overline{B o}$ (see Appendix A). The emission angle is again assumed to be zero, and $L=0.55$. The total RMSE has been shown in Fig. 7(b).

FIG. 17: Horizontal cuts along $\iota_{z}=12^{\circ}$ in Figs. 7 and 15-16. The bias and error due to small-scale albedo variability such that $\sigma_{B o}=0.005 \times \overline{B o}$, as well as the total bias and error in this case are also shown. 


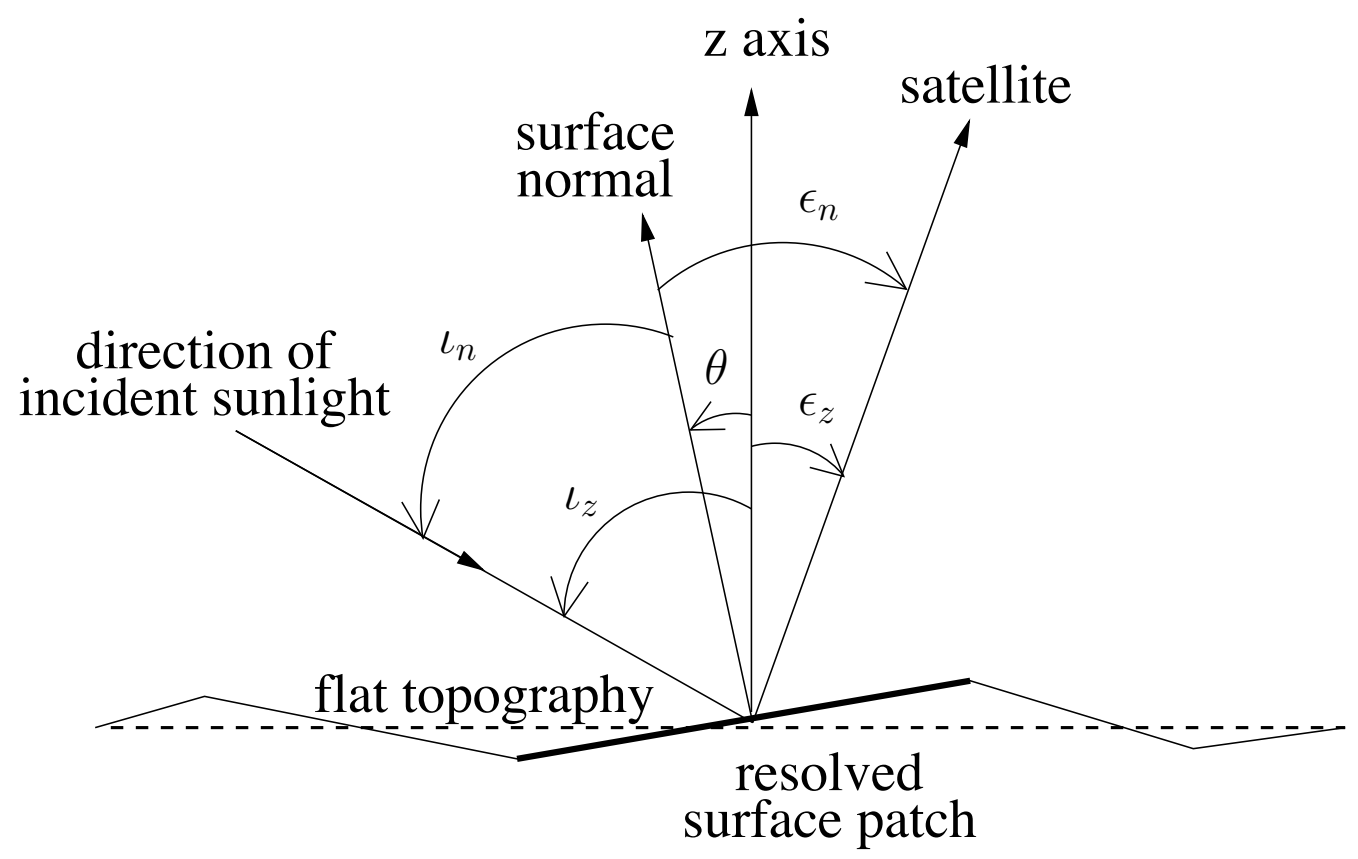

Figure 1: 